\title{
A STUDY OF RESEARCH NOTIONS IN WIRELESS BODY SENSOR NETWORK (WBSN)
}

\section{Dinesh Kumar,}

Computer Science and Engineering, Koneru Lakshmaiah Education Foundation, Andra Prdesh, India.

Email: adinesh@kluniversity.in
T. Kalaipriyan,

Computer Science and Engineering, Koneru Lakshmaiah Education Foundation, Andra Prdesh, India.

Email: kalaip27@gmail.com

\author{
R.S. Raghav, \\ Computer Science and Engineering, \\ Koneru Lakshmaiah Education Foundation, \\ Andra Prdesh, India. \\ Email: vpmrags@gmail.com
}

\begin{abstract}
The Body Sensor Networks is captivated in gathering the communication module in a more reliable manner along with efficiency in terms of energy, more secure than earlier schema's, ands enhanced utilization of resources. Wireless BAN (WBAN) has the tendency to be placed even inside the human body. The significance of BSN has the tendency to go through all aspects such as fitness of a person, his/her health issues, caring in critical level, and so on. In 2014 [1] the elevation of body sensors has been grown upto 420 millions from 11 million units in 2009. In this research work, we addressed BAN from On-Body to Body-to-Body cooperative networks at different levels: propagation, protocols and localization applications.
\end{abstract}

Keywords: Wireless Sensors, Body Sensors, Security and Research Trends.

\section{Introduction}

WSN has a wide range of applications including monitoring of environment in remote locations and tracking the targets in all aspects. The sensors of WSN will communicate with each other to form a network and to share or forward the information or collected data via it. In WSN, there are two different types of network with it, one is structured and the another in unstructured. In terms of structured network the sensors are planted in an effective pre-planned way. In unstructured network, the deployment of sensors will be in random manner [1]. Each sensor node has three basic components: a sensing subsystem, a processing system and communication system [2].

WSNs have been successfully applied to many industrial and civil domains, including industrial process, monitoring and control, environment and habitat monitoring, home automation, traffic control, machine health monitoring and healthcare applications, environmental monitoring, biological detection, industrial diagnostics [3], [4] also different types of sensors are useful to gather multiple parameters together, such as body temperature, blood pressure, pulse, heartbeat and blood sugar. The sensor network should have a lifetime long enough to fulfil the application requirements [5], [6]. 
J. Sustain. Wireless Syst.

Vol.01/ No. 02

Pages: $77-86$

http://irojournals.com/irosws/

DOI: https://doi.org/10.36548/jsws.2019.2.001

The development of Body Sensor Networks (BSN) has been facilitated by the rapid advances in Wireless Sensor Networks in recent years. Body Sensor Networks nodes provide a versatile environment for wireless sensing research and development. In addition to providing continuous monitoring and analysis of physiological parameters, the recently proposed the Body Sensor Networks incorporates context aware sensing for increased sensitivity and specificity [7]. BSNs can be wired (interconnecting with smart fabric) or wireless (making use of common wireless sensor networks and standards). Research in computer, networking, and medical fields are working together in order to make the broad vision of smart Body sensor network. After the introduction of dedicated BSN platforms, such as the BSN node, Pluto, Shimmer, purposely built platforms have been used in BSN research. [8]. The similar set of works can be found in [914].

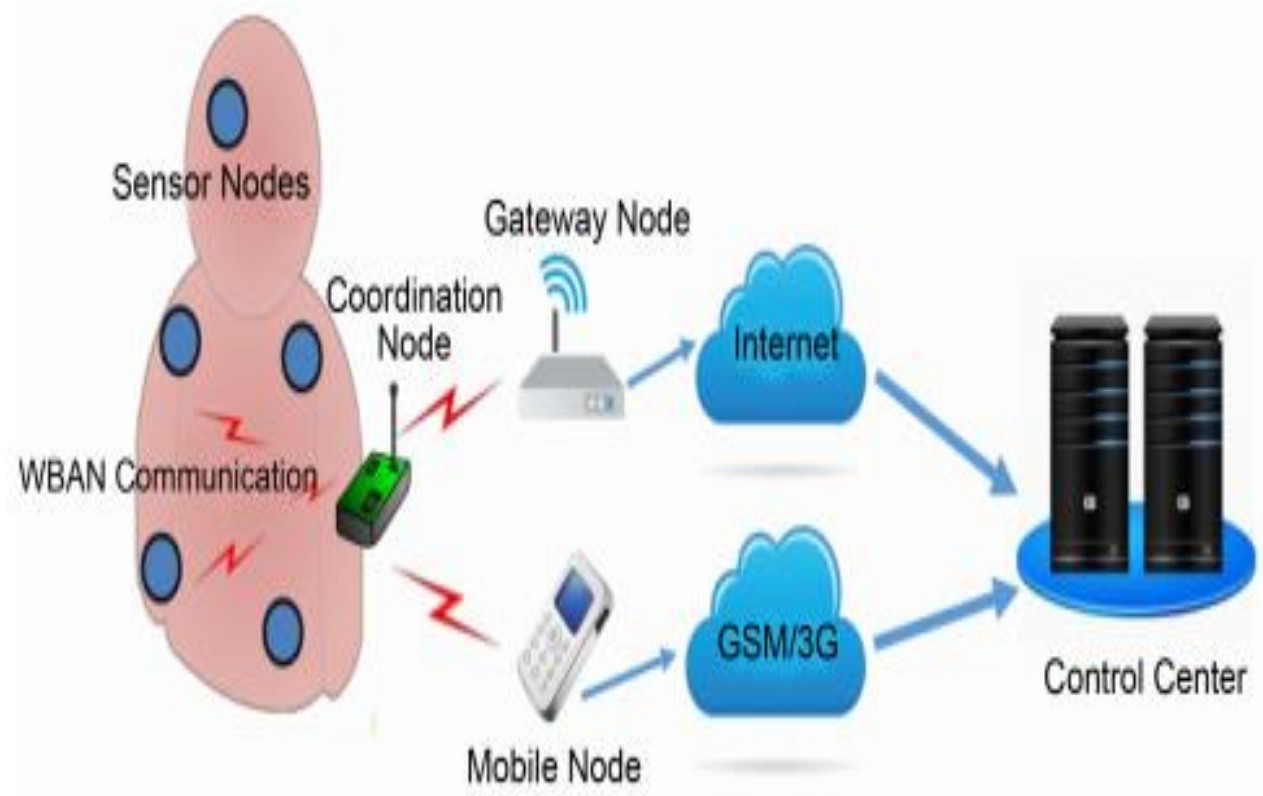

Figure 0.1 WBSN Architecture

\section{Related Work:}

Table.1 Summary of Literature Review in Related Works

Author

Title of the paper 
J. Sustain. Wireless Syst.

Vol.01/ No. 02

Pages: $77-86$

http://irojournals.com/irosws/

DOI: https://doi.org/10.36548/jsws.2019.2.001

\begin{tabular}{|c|c|c|}
\hline $\begin{array}{l}\text { JIN Mu-Jung } \\
\qquad \& \\
\text { QU Zhao-Wei [15] }\end{array}$ & $\begin{array}{l}\text { Optimised scheme of the } \\
\text { neighbour collaboration algorithm } \\
\text { SM-WSN. }\end{array}$ & $\begin{array}{l}\text { It has been deployed in a } \\
\text { number of fields including } \\
\text { monitoring of environments, } \\
\text { detection of problems in } \\
\text { biological sectors etc. }\end{array}$ \\
\hline Zhyan Xie [16] & $\begin{array}{l}\text { Clustering using WBAN } \\
\text { with clique-based method. }\end{array}$ & $\begin{array}{l}\text { A scheduling schema } \\
\text { based on the colors has been } \\
\text { proposed. }\end{array}$ \\
\hline Ousmane Diallo [17] & $\begin{array}{l}\text { Cloud based WBAN's with } \\
\text { statistical modelling techniques. }\end{array}$ & $\begin{array}{l}\text { For effective processing } \\
\text { of queries and to optimize the } \\
\text { overall storage infrastructure }\end{array}$ \\
\hline $\begin{array}{l}\text { Yena Kin } \\
\quad \& \\
\text { SuKyoung Lee }[18]\end{array}$ & $\begin{array}{l}\text { Maximises the Life time of } \\
\text { the whole integrated wireless hospital } \\
\text { sensor network. }\end{array}$ & $\begin{array}{l}\text { The theme of this } \\
\text { research is intended to enhance } \\
\text { the network with high efficiency } \\
\text { of power in large networks. }\end{array}$ \\
\hline Mehmet R Yuce [19] & $\begin{array}{rrr}\text { Wireless } & \text { Body } & \text { Area } \\
\text { Network System. } & & \\
\end{array}$ & $\begin{array}{l}\text { Designed for health care } \\
\text { applications. In the medical } \\
\text { stream a fully equipped body } \\
\text { sensor has been deployed. }\end{array}$ \\
\hline Steven Jovica Marinkovi [20] & $\begin{array}{l}\text { A novel- low power reliable } \\
\text { MAC protocol. }\end{array}$ & $\begin{array}{l}\text { Ihe outcome of this } \\
\text { research work states that this } \\
\text { proposed methodology along } \\
\text { with WBAN has the tendency to } \\
\text { work on EEG.. }\end{array}$ \\
\hline
\end{tabular}

\section{Methodology}

Wireless-body-area-networks (WBAN) is one of the network that has the tendency to collect the information's such as temperature of the body, blood pressure (BP), pulse, hearbeat of the person and the blood sugar level. This proposed methodology is intended to track the patients in these aspects in order to find the emergency level of any patient.

In this proposed methodology, an algorithm named SBWS and a technique on cloud namely WBAN has be hybridized. In the initial sector the SBWS, clustering will takes place where the set of sensors will be grouped in order to avoid the overlap. In this method scheduling method which works based on the priority has been imposed. The gathered information will then be processed through cloud network for effective processing of required queries. 
J. Sustain. Wireless Syst.

Vol.01/ No. 02

Pages: $77-86$

http://irojournals.com/irosws/

DOI: https://doi.org/10.36548/jsws.2019.2.001

In the second phase of the proposed system, a real clpoud structure based on the storage and optimization on processing of queries will be placed in order to minimize the overall energy and to reduce the query latency. An effective Contiguous Link scheduling algorithm has been imposed for effective processing of queries.

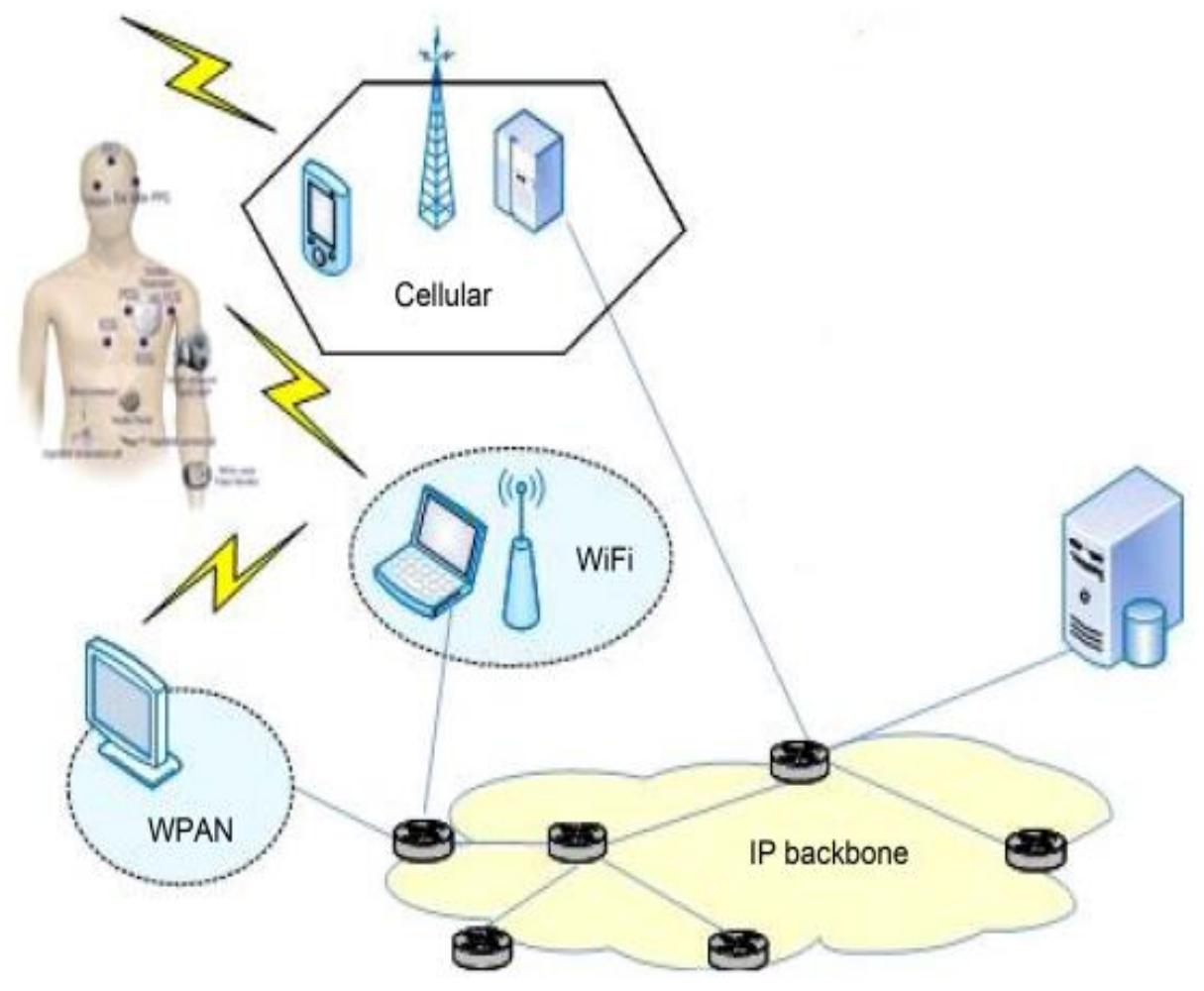

Figure 3. A Typical WBSN communication

Table 0.1

Physiological signals range by sensors in WBAN

\begin{tabular}{|c|c|}
\hline Physiological Signal & Signal frequency range/Bandwidth (Hz) \\
\hline ECG signal & $0.01-250$ \\
\hline Blood pressure (BP) & $0-50$ \\
\hline EEG & $0.5-60$ \\
& $0-0.1$ \\
\hline Body temperature & $<0.1$ \\
\hline pulse & $30-235 \mathrm{bpm}$ \\
\hline blood oxygen levels &
\end{tabular}


J. Sustain. Wireless Syst.

Vol.01/ No. 02

Pages: $77-86$

http://irojournals.com/irosws/

DOI: https://doi.org/10.36548/jsws.2019.2.001

Table $0.2 \quad$ Technical requirements

\begin{tabular}{|l|l|}
\hline Parameters & Specifications \\
\hline Communication Medium & Wireless channel \\
\hline Communication protocol & MAC (Media Access Control) \\
\hline Modulation/coding techniques & FSK (Frequency-Shift Key) \\
\hline Frequency band specification & $10 \mathrm{kHz}-1 \mathrm{MHz}$ \\
\hline Topology information & Star topology \\
\hline Attenuation & 0.625 volts \\
\hline SNR & $16 \mathrm{db}$ \\
\hline Layer & PHY layer, MAC layer and Network layer \\
\hline
\end{tabular}

\section{Distributed Local Clique Finding Algorithm}

In DLCF algorithm the neighborhood-based cliques will be found using n-clique method. On the off chance that all sensors of n-Clique originate from a solitary WBAN, we can discover the nearby Clique effortlessly by the sensors' identifier. Something else, if the sensors of t-Clique originate from various diverse Cliques, we discovered all the nearby Cliques by finding neighborhood Clique Algorithm.

By and large, we can present an answer to the issue of discovering the t-Clique in an appropriate manner as takes after. We proposed a methodology that possess the data table in each of it, and the ID's of neighbor sensors. Also, a set of $\mathrm{k}$ sensors are passed in WBAN to pass body maladies. Then DLNF algorithm is used to find local n-Clique. For each sensor, say $\mathrm{t} 1$ does as the following. 
J. Sustain. Wireless Syst.

Vol.01/ No. 02

Pages: $77-86$

http://irojournals.com/irosws/

DOI: https://doi.org/10.36548/jsws.2019.2.001

Step 1 Assume $t_{2}, t_{3}, \ldots, t_{s}$ be all the active neighbors of $t_{1}$ and $t^{\prime}{ }_{1}, t^{\prime}{ }_{2}, \ldots, t^{\prime}{ }_{s}$ represent the IDs of $t_{1}, t_{2}, \ldots, t_{s}$ respectively. Let $M_{1}, M_{2}, \ldots, M_{s}$ represent $N S L N$ and $M^{\prime}{ }_{1}$, $M_{2}^{\prime}, \ldots, M_{s}^{\prime}$ represent IDNS NSLN of respectively.

Step 2 For $(n=s ; n \geq k ; t--)$ do

$t_{1}$ compute different subsets $S_{1}, S_{2}, \ldots \ldots$, of

Let represent the set of all the $y$-clique $(n<Y \leq s)$ that have been found by node represent the node sets of a y-Clique in.

For

IF there is no such that

Let

Let $C=$

If record $C$ as a $n$-Clique end IF

End for

End for

\section{Experimental Results}

In this section the overall simulation results of the proposed method and its mechanism of query with optimization in the real time has been evaluated. In our research, the actual sensors have the tendency to group in different manner in order to eliminate interference by flagging it with different colors. And these scheduled sensors will be secured and used it in the real time environment. By using these techniques, we achieved high throughput, low query latency and less energy consumption.

SOFTWARE: MATLAB

Version : MATLAB 8.1 R2013a

HARDWARE:

OS: Windows 10

PROCESSOR: Intel®Pentium®CPU G2030 @ 3.00GHz

RAM: 4.00GB

SYSTEM TYPE: 64-bit OS

\subsection{Throughput}

The throughput can be defined as an actual transmission per slot (TPS) which calculates data transmissions of all sensors in the system and is the performance index used to estimate WBAN scheduling. We compare the system throughput of our DLCF method with CPN-based RIC in small, medium, high, and very high WBAN densities. 
J. Sustain. Wireless Syst.

Vol.01/ No. 02

Pages: $77-86$

http://irojournals.com/irosws/

DOI: https://doi.org/10.36548/jsws.2019.2.001

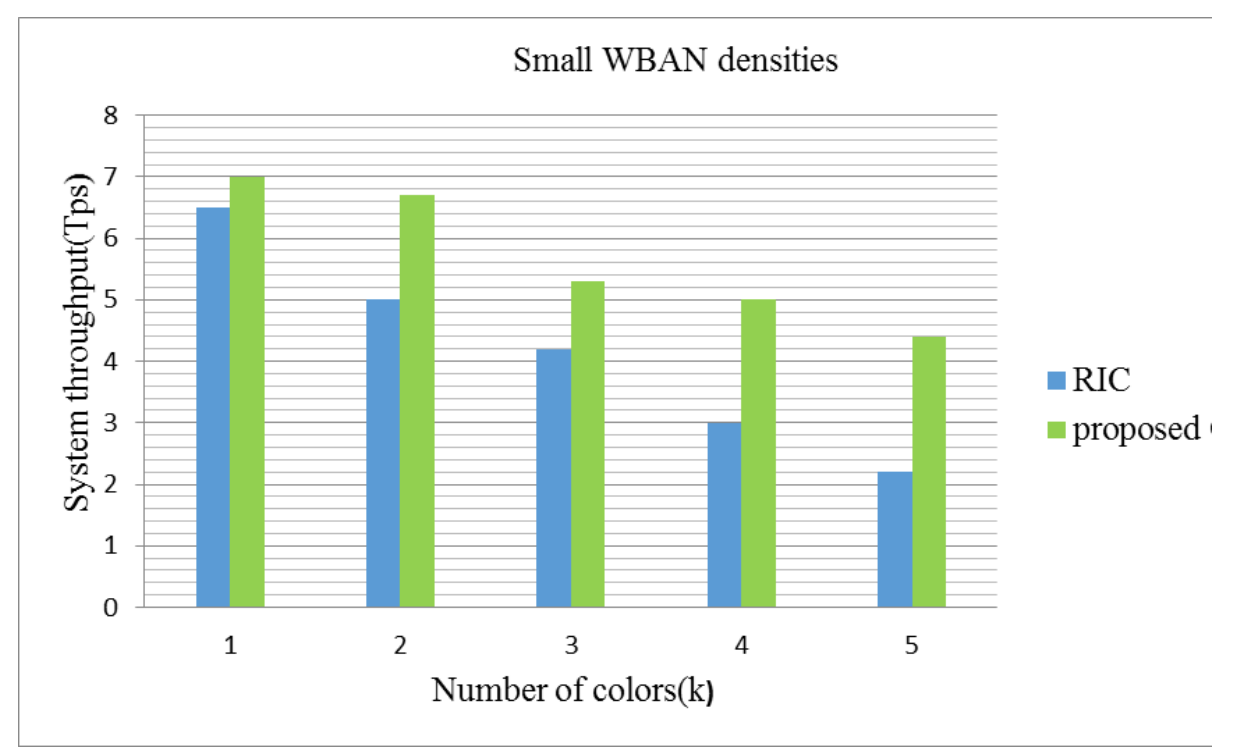

Fig.2 Throughput of WBAN scheduling system with low density

Figure 2 shows that the system throughput decreases along with the increase in total sum of the colors since the scheduling process will be increased with the increase in number of colors. By compared to the worst case our proposed DLCF is better in small WBAN densities.

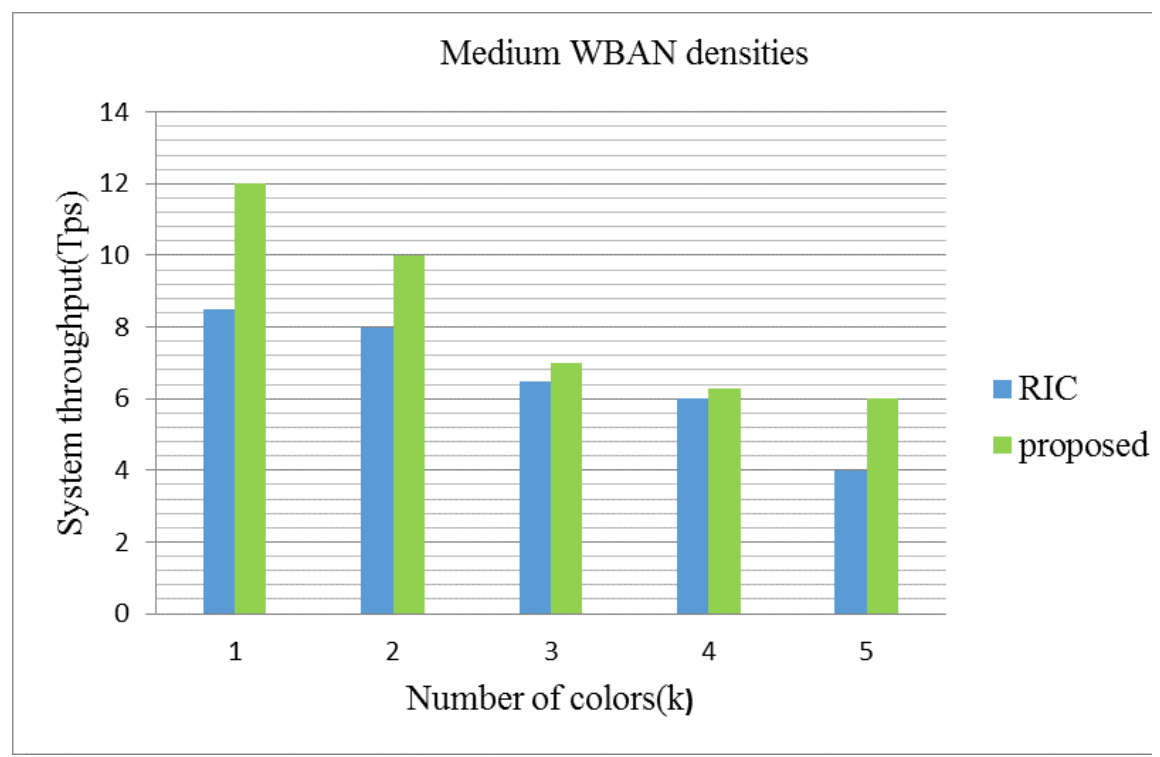

Fig.3 Throughput of WBAN scheduling system with medium density 
J. Sustain. Wireless Syst.

Vol.01/ No. 02

Pages: $77-86$

http://irojournals.com/irosws/

DOI: https://doi.org/10.36548/jsws.2019.2.001

Figure 3 show that the system throughput is slightly decreases along with the increase in total sum of the colors since the scheduling process will be increased with the increase in number of colors. By compared to the worst case our proposed DLCF is better in medium WBAN densities.

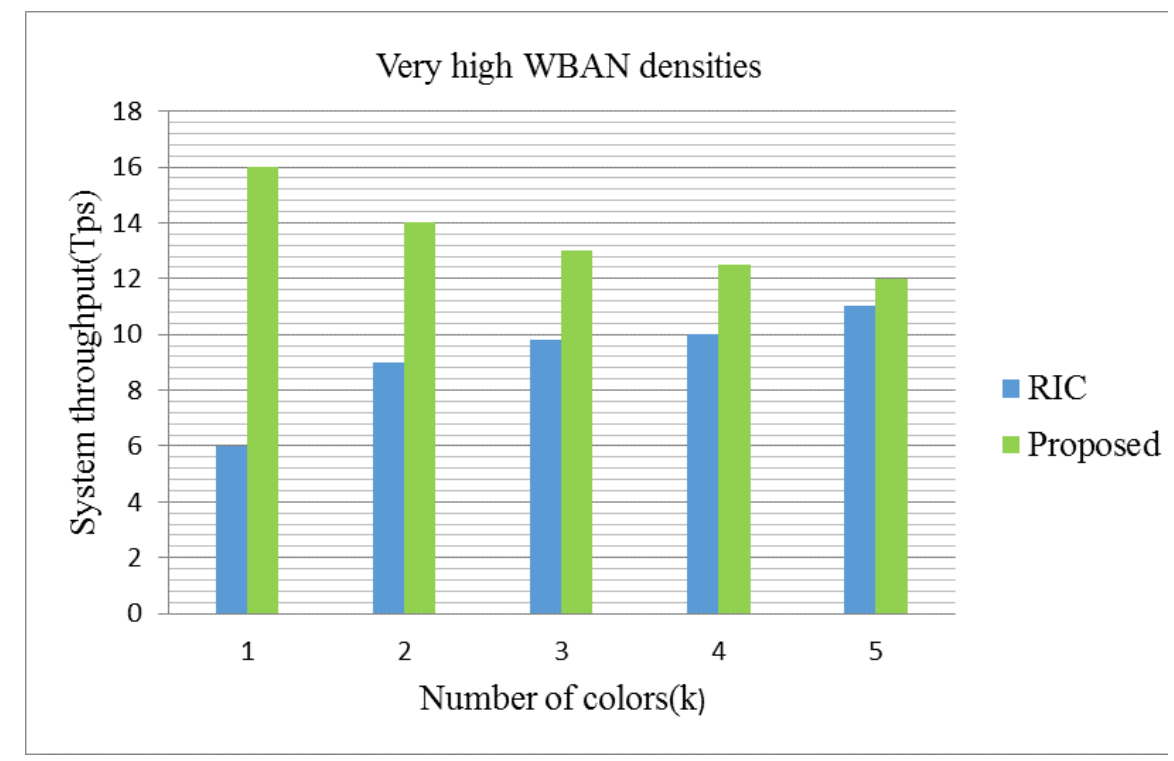

Fig.5 Throughput of WBAN scheduling system with very high density

Figure 5 show that the system throughput is tremendously increases along with the increase in total sum of the colors since the scheduling process will be increased with the increase in number of colors. By compared to the worst case our proposed DLCF is better in highly WBAN densities.

\section{Conclusion}

In this paper we proposed a clique-based scheduling algorithm and cloud-based scheduling algorithm. In clique-based technique, the sensors can be clustered in to groups to avoid interference to Distributed Local Clique Finding In cloudbased technique, the stored information should be secure and also used it in the real time environment which demands to use Multi Queue Scheduling. By combining these two techniques we achieved better throughput, energy consumption and query latency compared to the previous cases and also it will be optimized in real time environment.

\section{References:}

[1] JenniferYick, Biswanath Mukherjee and Dipak Ghosal, "Wireless sensor network survey", Computer networks, Vol. 52, No. 12, pp. 2292-2330, 2008.

[2] I.F. Akyildiz, W. Su, Y. Sankarasubramaniam and E. Cayirci,"Wireless sensor networks: a survey", Computer networks, Vol. 38, No. 4, pp. 393-422, 2002. 
J. Sustain. Wireless Syst.

Vol.01/ No. 02

Pages: $77-86$

http://irojournals.com/irosws/

DOI: https://doi.org/10.36548/jsws.2019.2.001

[3] JIN Mu-jing and Zhao-wei QU, "Efficient neighbor collaboration fault detection in WSN", The Journal of China Universities of Posts and Telecommunications, Vol. 18, pp. 118-121, 2011.

[4] Miao Xie, Song Han, Biming Tian and Sazia Parvin, "Anomaly detection in wireless sensor networks: A survey", Journal of Network and ComputerApplications, Vol. 34, No. 4, pp. 1302-1325, 2011.

[5] Giuseppe Anastasi, Marco Conti, Mario Di Francesco and Andrea Passarella, "Energy conservation in wireless sensor networks: A survey", Ad hoc networks Vol. 7, No. 3, pp. 537-568, 2009.

[6] Zhijun Xie, Guangyan Huang, Jing He and Yanchun Zhang, "A clique-based wban scheduling for mobile wireless body area networks", Procedia Computer Science, Vol. 31, pp. 1092-1101, 2014.

[7] Hande Alemdar and Cem Ersoy, "Wireless sensor networks for healthcare: A survey", Computer Networks, Vol. 54, No. 15, pp. 2688-2710, 2010.

[8] Benny Lo and Guang-Zhong Yang, "Wireless Sensor Development Platforms", Body Sensor Networks, pp. 527-564, 2014.

[9] Bandar Alghamdi and Hacène Fouchal, "A mobile wireless body area network platform", Journal of Computational Science, Vol. 5, No. 4, pp. 664-674, 2014.

[10] Mehmet R. Yuce, "Implementation of wireless body area networks for healthcare systems", Sensors and Actuators A: Physical, Vol. 162, No. 1, pp. 116-129, 2010.

[11]Jocelyne Elias and Ahmed Mehaoua, "Energy-aware topology design for wireless body area networks", Communications (ICC), IEEE International Conference, pp. 3409-3413, 2012.

[12] Ousmane Diallo, Joel J.P.C. Rodrigues, Mbaye Sene and Jianwei Niu, "Real-time query processing optimization for cloud-based wireless body area networks", Information Sciences, Vol. 284, pp. 84-94, 2014.

[13] Saeideh Sadat Javadi and M. A. Razzaque, "Security and privacy in wireless body area networks for health care applications", Wireless Networks and Security, Springer Berlin Heidelberg, pp.165-187, 2013.

[14] Ming Li, Wenjing Lou, and Kui Ren, "Data security and privacy in wireless body area networks", IEEE Wireless Communications, Vol. 17, No. 1, pp. 51-58, 2010.

[15] JIN Mu-jing and Zhao-wei QU, "Efficient neighbor collaboration fault detection in WSN", The Journal of China Universities of Posts and Telecommunications, Vol. 18, pp. 118-121, 2011.

[16]Zhijun Xie, Guangyan Huang, Jing He and Yanchun Zhang, "A clique-based wban scheduling for mobile wireless body area networks", Procedia Computer Science, Vol. 31, pp. 1092-1101, 2014.

[17] Ousmane Diallo, Joel J.P.C. Rodrigues, Mbaye Sene and Jianwei Niu, "Real-time query processing optimization for cloud-based wireless body area networks", Information Sciences, Vol. 284, pp. 84-94, 2014.

[18] Yena Kim and SuKyoung Lee, "Energy-efficient wireless hospital sensor networking for remote patient monitoring", Information Sciences, Vol. 282, pp. 332-349

[19] Mehmet R Yuce, "Implementation of wireless body area networks for healthcare systems", Sensors and Actuators A: Physical, Vol. 162, No. 1, pp. 116-129, 2010.

[20] Stevan Jovica Marinkovic, Emanuel Mihai Popovici, Christian Spagnol, Stephen Faul and William Peter 
J. Sustain. Wireless Syst.

Vol.01/ No. 02

Pages: $77-86$

http://irojournals.com/irosws/

DOI: https://doi.org/10.36548/jsws.2019.2.001

Marnane, "Energy-efficient low duty cycle MAC protocol for wireless body area networks", IEEE Transactions Information Technology in Biomedicine, Vol. 13, No. 6, pp. 915-925, 2009. 